EPJ Web of Conferences 73, 04013 (2014)

DOI: $10.1051 /$ epjconf/20147304013

(C) Owned by the authors, published by EDP Sciences, 2014

\title{
The double polarization program of CBELSA/TAPS
}

Annika Thiel ${ }^{\mathrm{a}}$ for the CBELSA/TAPS collaboration

Helmholtz-Institut für Strahlen- und Kernphysik, Nussallee 14-16, 53115 Bonn, Germany

\begin{abstract}
The excitation spectrum of the proton consists of resonances with substancial width which are often strongly overlapping and are therefore difficult to disentangle. To determine the exact contributions and identify these resonances, a partial wave analysis solution has to be found. For a complete experiment, which leads to an unambiguous solution, several single and double polarization observables are needed. With the Crystal Barrel/TAPS experiment at ELSA, the measurement of double polarization observables in different reactions is possible by using a circularly or linearly polarized photon beam on a transversely or longitudinally polarized butanol target.
\end{abstract}

\section{Introduction}

For the nucleon excitation spectrum several theoretical predictions are available. Aside from constituent quark models, first lattice QCD calculations have now been conducted. In Fig. 1 predictions by the Bonn Model [1] and the more recent lattice QCD calculations by Edwards et al. [2] are shown. Even if the lattice QCD calculations are performed at a non-physical pion mass, the correct level counting of the states is already visible. In both models states have been predicted, which were not confirmed so far and the most promising search can be done using photoproduction.

The resonant states have a large width and overlap, see Fig. 1. For the extraction of these resonances from the data, partial wave analyses have to be performed, which need a preferably complete database of polarization observables as input.

\section{Experimental setup}

The setup of the CBELSA/TAPS experiment, which is located at the ELSA electron accelerator in Bonn, is shown in Fig. 2. Photons are created from electrons via bremsstrahlung at different radiator targets to produce linearly or circularly polarized photons. Several different reaction targets are available, for example longitudinally or transverely polarizable butanol targets, a liquid hydrogen target or carbon targets for background studies. The according target is surrounded by the Crystal Barrel detector, consisting of $1320 \mathrm{CsI}(\mathrm{Tl})$ crystals. In forward direction the angular coverage is closed by the MiniTAPS

\footnotetext{
ae-mail: thiel@hiskp.uni-bonn.de
}

This is an Open Access article distributed under the terms of the Creative Commons Attribution License 4.0, which permits unrestricted use, distribution, and reproduction in any medium, provided the original work is properly cited. 

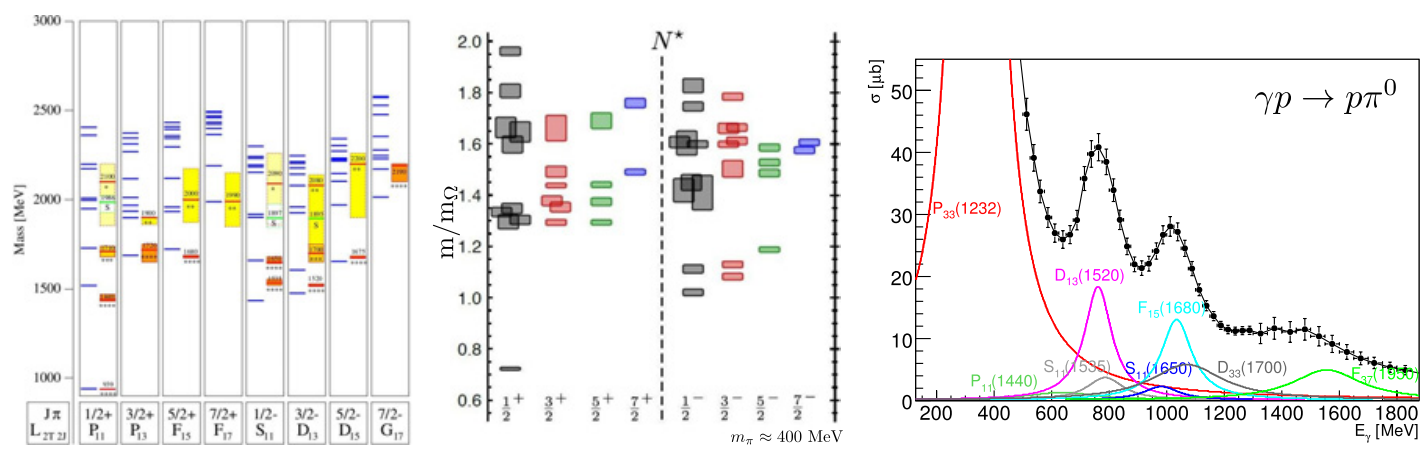

Figure 1. Left: measured and predicted resonances of a relativistic quark model [1]. Center: predictions of baryon resonances by lattice QCD calculations [2] Right: the cross section of $\pi^{0}$ photoproduction, shown are also the calculated Breit-Wigner distributions of the different contributing resonances.

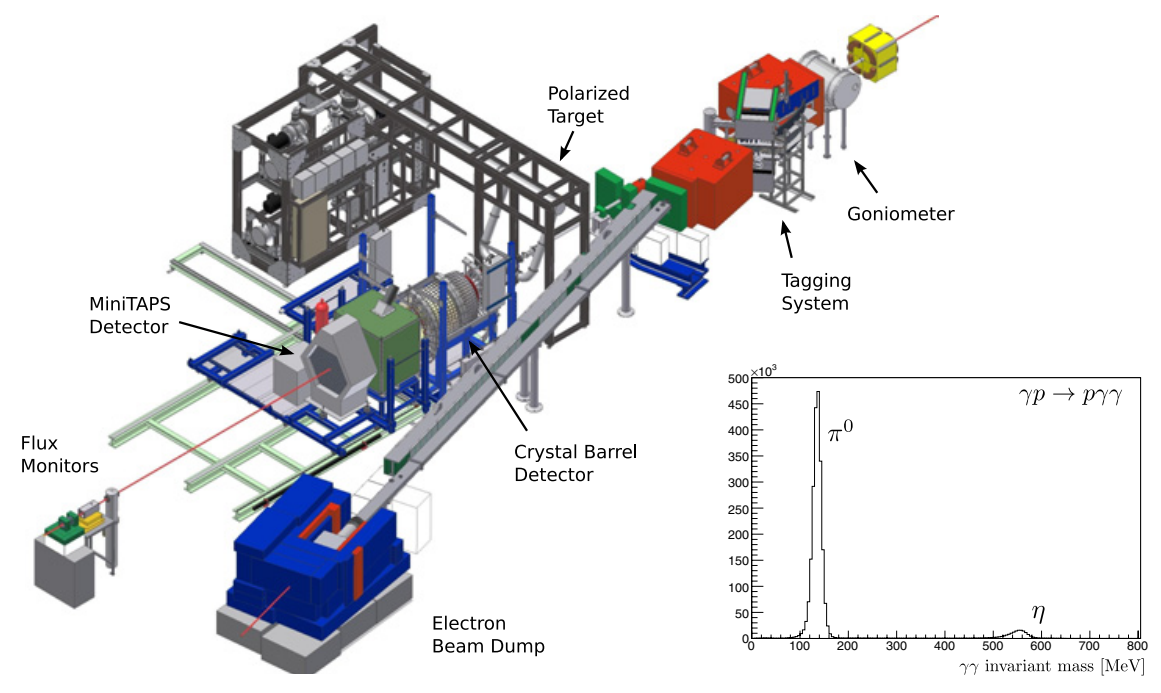

Figure 2. The experimental setup of the CBELSA/TAPS experiment at ELSA. Inset: the invariant mass of two photons with the $\pi^{0}$ and $\eta$ meson.

detector with $216 \mathrm{BaF}_{2}$ crystals, which covers the polar angle down to $1^{\circ}$. In front of all calorimeters are charge sensitive detectors installed.

The complete setup offers nearly full $4 \pi$ angular coverage with a high detection efficiency for neutral particles. It is therefore ideally suited to measure polarization observables with neutral final states.

\section{The selection process and extraction of the polarization observables}

To select the desired final state, different cuts are possible. First, reactions with two neutral hits were selected. If a third hit has been found, it has to be charged and was attributed to the proton. Besides a cut on the time difference of the detected particles, a constraint on the kinematics of the decay process was invoked by analyzing the $\theta$ and $\phi$ differences between meson and recoiling proton. The proton was treated as a missing particle, so that a further constraint could be set on the calculated mass of the 

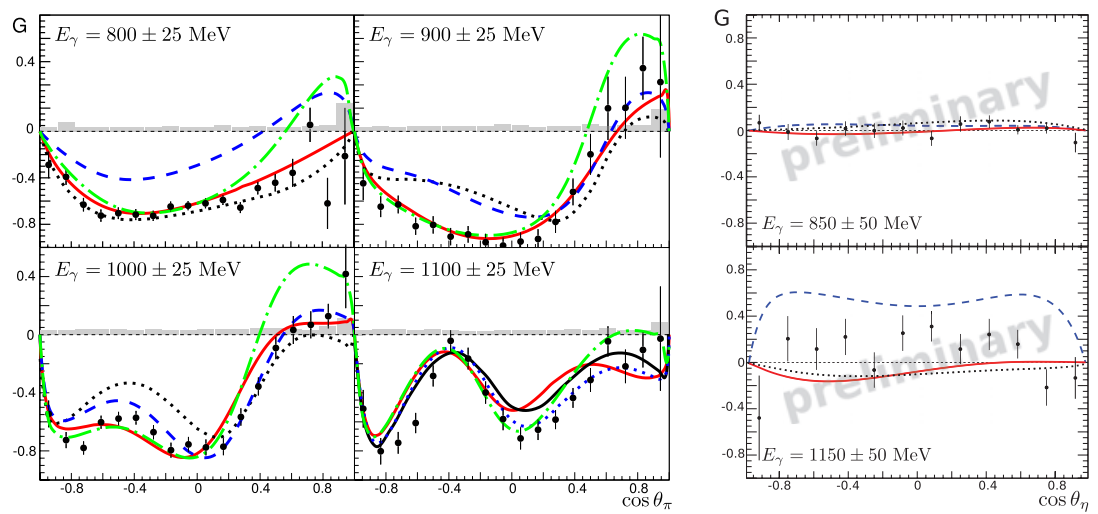

Figure 3. Results for the double polarization observable $G$ in $\pi^{0}$ (left) [7] and $\eta$ (right) [6] photoproduction, compared to predictions by the MAID (black, dotted line) [3], SAID (SN11: blue dashed line, CM12: green, dashed dotted line) [4] and BnGa (red, solid line) [5] analysis.

proton. With these selection rules, the $p \pi^{0}$ and $p \eta$ final state could successfully be extracted with nearly no background, as can be seen in Fig. 2.

By using a longitudinally polarized butanol target and a linearly polarized photon beam, the cross section of pseudoscalar meson photoproduction is given by

$$
\frac{d \sigma}{d \Omega}\left(\theta_{\pi}, \phi_{\pi}\right)=\left.\frac{d \sigma}{d \Omega}\right|_{0} \cdot\left(1-p_{\gamma} \Sigma \cos \left(2 \phi_{\pi}\right)+p_{\text {lin }} p_{T} G \sin \left(2 \phi_{\pi}\right)+p_{\text {circ }} p_{T} E\right)
$$

with the double polarization observables $G$ and $E$, the beam asymmetry $\Sigma$ and the polarization degree of the protons $\left(p_{T}\right)$ and the linearly $\left(p_{\text {lin }}\right)$ or circularly $\left(p_{\text {circ }}\right)$ polarized photons.

Due to carbon bound in the butanol molecule, it is necessary to determine the contribution of reactions off unpolarized carbon in the data. This is done by using measurements with a foam carbon target $\left(N_{C}\right)$ and comparing it to the data on the butanol target $\left(N_{B}\right)$. A dilution factor $d=1-\frac{N_{C}}{N_{B}}$ was extracted and used to correct the measured values. The double polarization observable $G$ was therefore successfully determined by analyzing the $\phi$ distributions of the measured meson. The distributions of the observable are shown for the $p \pi^{0}$ and $p \eta$ final state in Fig. 3. In the $p \pi^{0}$ final state, differences of the MAID and SAID predictions to the data occur already in the second resonance region $\left(E_{\gamma} \approx 800 \mathrm{MeV}\right)$. These differences were investigated and could be attributed to the $E_{0+}$ and $E_{2-}$ multipole [7]. Using a new fit method by the SAID group leads to a better description of the data [8]. For $\eta$ photoproduction neither of the predictions describe the data points well. Especially at higher energies huge differences become visible, which indicate that there are still unkown processes involved in the $p \eta$ final state.

With a circularly polarized photon beam impinging on a longitudinally polarized butanol target, the double polarization observable $E$ can be measured. The observable is a helicity asymmetry between both states where nucleon and photon spin are either parallel or antiparallel aligned. As an example, four energy bins of this observable are shown in Fig. 4. It becomes obvious, that especially at high energies huge differences between the data and the predictions exist [9]. Here it is necessary to include the newly extracted data in the different PWA fits to constrain the solutions in order to describe the data points.

\section{Interpretation and conclusion}

The newly extracted observables on $\pi^{0}$ photoproduction $\mathrm{E}$ and $\mathrm{G}$, with new data on $\mathrm{T}, \mathrm{P}$ and $\mathrm{H}$ [10], have been included in the BnGa partial wave analysis. The preliminary results for the real and imaginary 


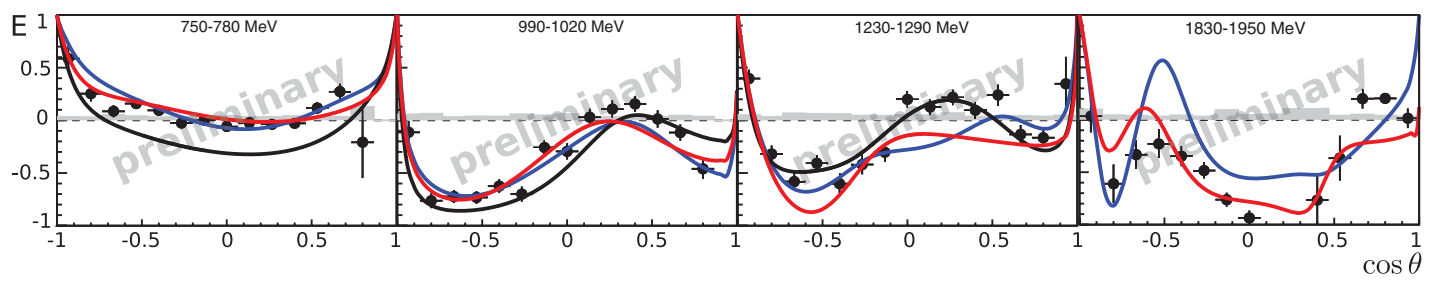

Figure 4. Four different energy bins of the double polarization observable $E$ in $\pi^{0}$ photoproduction [9] compared to predictions by the MAID (black) [3], SAID (blue) [4] and BnGa (red line) [5] analysis.
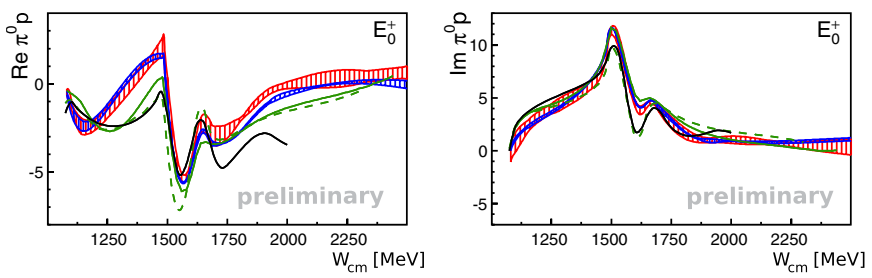

Figure 5. Results of the multipoles for a preliminary fit of the BnGa PWA [5] (blue) compared to the old solution (red) and different solutions by MAID (black) [3] and SAID (green solid SN11, green dashed CM12) [4].

part of the $E_{0+}$ multipole are shown in Fig. 5. Due to this multipole, to which e.g. the $S_{11}$ resonances contribute, huge differences have been observed in the polarization observable $G$ [7]. Fitting the new polarization observables constrains the fit further and the error is reduced. Including these data sets in the other PWAs will hopefully lead to a more unique solution for the multipoles and therefore help to determine the resonance parameters. The new observables measured at ELSA and other laboratories will pave the way to a better understanding of the nucleovn excitation spectrum.

This work was supported by the Deutsche Forschungsgemeinschaft (DFG) within SFB/TR16.

\section{References}

[1] U. Loering et al., Eur. Phys. J. A 10, 395-446 (2001)

[2] R. Edwards et al., Phys. Rev. D 84, 074508 (2011)

[3] MAID http://wwwkph.kph.uni-mainz.de/MAID

[4] SAID http://gwdac.phys.gwu.edu

[5] BnGa http://pwa.hiskp.uni-bonn.de

[6] M. Grüner, [arXiv: 1109.4813]

[7] A.Thiel et al., Phys. Rev. Lett. 109, 102001 (2012)

[8] R. Workman et al., Phys. Rev. Lett. 110, 169101 (2013); A.Thiel et al., Phys. Rev. Lett. 110, 169102 (2013)

[9] M. Gottschall et al., Phys. Rev. Lett. 112, 012003 (2014)

[10] J. Hartmann et al., publication in preparation 